\title{
ANALYSIS ON 85 CASE REPORTS OF ADVERSE DRUG REACTIONS
}

\author{
Xiang-qiu Jin , Lian-qiu Min* \\ The First Affiliated Hospital of Liaoning Medical College, Jinzhou 121001, Liaoning Province, China. \\ *E-mail: jy4197092@yeah.net
}

\begin{abstract}
This study investigated the occurrence and characteristics of adverse drug reactions (ADR) in our hospital and provide references for clinical rational drug use. We collected the 85 case reports of adverse drug reactions in our hospital in 2010 and made retrospective statistical analysis on them. The varieties of anti-infective drugs used are the most used. It also had the highest proportion of adverse drug reactions; the common symptom of adverse drug reactions is skin and accessory damage. Adverse drug reactions are affected by many factors, and relevant departments should strengthen ADR monitoring, to reduce or avoid the occurrence of adverse drug reactions.
\end{abstract}

Key words: adverse drug reaction; monitoring; rational drug use

\section{Introduction}

Adverse drug reactions (ADR) refer to the harmful reactions unrelated with drug use purposes caused by approved drugs under normal usage and dosage, including side effects, allergic reactions, toxic reactions, drug dependence, mutation, distortion, canceration, etc. With the continuous and deepened implementation of ADR monitoring system in our country, ADR incidence continues to increase. In order to improve body physiology, improve the quality of clinical treatment and reduce medical disputes, ADR monitoring and reporting have become the legal obligations for medical institutions (Jin et al., 2004). With the continuous strengthening of drug use management, ADR monitoring becomes increasingly important to guaranteeing public drug use safety, and strengthening. ADR monitoring function has become an important content of the current epidemiological studies on drugs (Zhao et al., 2009). This paper assorts and analyzes the 85 ADR cases in our hospital reported in 2010 and summarizes the occurrence and characteristics of adverse reactions, providing scientific references for clinical safe, rational effective drug use.

\section{Data and methods}

\section{Data resource}

A total of 85 effective reports of ADR were reported by all the Departments in our hospital in 2010 . These were collected physicians and pharmacists who filled in the "Adverse drug reactions report form", and the overall ADR summary was made after the preliminary analysis and evaluation by clinical pharmacists.

\section{Methods}

A retrospective analysis was made on the clinical data of the 85 cases of ADR, to summarize ADR patients' gender and age distribution, routes of drug administration and clinical manifestations. Incidence of ADR caused by monotherapy and combination therapy, and ADR treatment outcomes were also analysed. 


\section{Results}

\section{Gender and ages of the patients with ADR}

In the 85 cases of ADR, 34 cases are male (34/85, 40\%), 51 cases are female $(51 / 85,60 \%)$, male to female ratio is about $0.67: 1$, and the incidence of ADR in females is higher than that in males. The patients are between 6 months -87 years old, and the population less than 20 years old is mostly pediatric patients $(6 / 22,27.27 \%)$. The age group with the highest ADR incidence is bellow 10 years old $(22 / 85,25.88 \%)$, followed by the age group of above 70 years old $(20 / 85,23.53 \%)$. The distribution of gender and ages of the patients with ADR is shown in Table 1.

Table 1: Distribution of gender and ages of the patients with ADR

\begin{tabular}{|c|c|c|c|c|c|c|c|c|c|}
\hline Gender & & & & Age & & & & & Total \\
\hline & $\leq 10$ & $11 \sim 19$ & $20 \sim 29$ & $30 \sim 39$ & $40 \sim 49$ & $50 \sim 59$ & $60 \sim 69$ & $\geq 70$ & \\
\hline Male & 8 & 2 & 4 & 1 & 3 & 2 & 5 & 9 & 34 \\
\hline Female & 14 & 6 & 4 & 3 & 4 & 3 & 6 & 11 & 51 \\
\hline Total & 22 & 8 & 8 & 3 & 7 & 5 & 11 & 20 & 85 \\
\hline Proportion (\%) & 25.88 & 9.41 & 9.41 & 4.71 & 8.24 & 5.88 & 12.94 & 23.53 & 100 \\
\hline
\end{tabular}

\section{Family ADR history and past ADR history}

In the 85 cases of ADR, 3 cases had family allergic history (3/85, 3.53\%), and 48 cases were unknown (46/85, 54.12\%); 18 cases had past ADR history (18/85, 21.18\%), and 51 cases were unknown (51/85, 60.00\%). The relationship between family and past ADR history and ADR is shown in Table 2.

Table 2: Relationship between family and past ADR history and ADR

\begin{tabular}{|c|c|c|c|c|c|}
\hline FamilyADR history & $\mathrm{n}$ & Proportion $(\%)$ & Past ADR history & $\mathrm{n}$ & Proportion $(\%)$ \\
\hline $\mathrm{Y}$ & 3 & 3.53 & $\mathrm{Y}$ & 18 & 21.18 \\
\hline $\mathrm{N}$ & 36 & 42.35 & $\mathrm{~N}$ & 16 & 18.82 \\
\hline Unclear & 46 & 54.12 & Unclear & 51 & 60.00 \\
\hline Total & 85 & 100.00 & Total & 85 & 100.00 \\
\hline
\end{tabular}

Distribution of drug administration routes causing ADR

The 85 cases of ADR involve a total of 3 drug administration routes, including 73 cases of intravenous infusion (73/85, 
http://dx.doi.org/10.4314/ajtcam.v10i3.18

$85.88 \%), 8$ cases of oral administration (8/85, 9.41\%), 3 cases of intramuscular injection $(3 / 853.53 \%)$ and 1 case of external use $(1 / 85,1.18 \%)$. The relationship between different drug administration routes and ADR is shown in Table 3 .

Table 3: Distribution of drug administration routes causing ADR

\begin{tabular}{lcl}
\hline Drug administration route & $\mathrm{n}$ & Proportion $(\%)$ \\
\hline Intravenous infusion & 73 & 85.88 \\
Oral administration & 8 & 9.41 \\
Intramuscular injection & 3 & 3.53 \\
External use & 1 & 1.18 \\
Total & 85 & 100.00 \\
\hline
\end{tabular}

Relationship between combination therapy and ADR incidence

The 85 cases of ADR include 68 cases of monotherapy $(68 / 85,80.00 \%), 17$ cases of combination therapy $(17 / 85$, $20.00 \%)$, and 12 cases involving antimicrobial drugs (12/85, 14.12\%). 16 cases were combination therapy of two drugs $(16 / 85,18.82 \%)$. The ratio of ADR incidence caused by monotherapy to combination therapy is $4: 1$.

\section{Drug varieties causing ADR and incidence}

We make the classification statistics on the drugs involved by ADR according to the drug classification method of Chinese Pharmacopoeia Commission (Chinese Pharmacopoeia Commission., 2001). The 85 cases of ADR involve a total of 50 drugs in 12 categories, in which, 65 cases of ADR were caused by antibiotics, accounting for the largest proportion (65/85, $76.47 \%$ ), including 1 case of severe allergic shock, and the others were mostly rash and other allergic-like reactions. In the cases of ADR, the varieties ranked in decreasing order: amoxicillin sulbactam sodium, ceftriaxone and levofloxacin injection.

\section{Prognosis}

All the symptoms of ADR relieve or disappear after drug withdrawal or changing/replacing for other treatment, and there was no case report of death.

\section{Discussions}

\section{Relationship between ages and gender of patients and ADR}

It can be seen from Table 1 that in the 85 cases of ADR in our hospital, the incidence in female is slightly higher than that in male, and they can occur at any age group. The proportion in children below 10 years old and elderly above 60 years old is significantly higher than other age groups, which may be related with the physiological characteristics of the elderly and children (Xie et al., 2004). For the elderly, because of visceral dysfunction, plasma and drug binding capability decreases, and different renal clearance rates result in different ADR incidence and severity. For children, underdevelopment of systems and organs and incomplete development of nervous system could be ascribed to the cause of ADR. In the 85 cases of ADR reports, 31 cases were above 60 years old, accounting for $36.47 \%$ of the total. The reasons are that the elderly have many 
chronic diseases, the frequency and quantity of drug use are higher than the youth and adults, and also the physiological functions and activities of the systems and drug metabolism capacity in the elderly begin to weaken (Jian et al., 2003). Four reasons make elderly patients prone to ADR (Xie et al., 2004): (1) With age increases, the organs in the elderly degrade year

Table 4: Drug varieties causing ADR and incidence

\begin{tabular}{|c|c|c|c|c|c|}
\hline Drug variety & $\begin{array}{l}\text { Generic name } \\
\text { (trade name) }\end{array}$ & $\mathrm{n}$ & $\begin{array}{l}\text { Proportion } \\
\qquad(\%)\end{array}$ & $\begin{array}{l}\text { Proportion of } \\
\text { variety }(\%)\end{array}$ & \\
\hline $\begin{array}{l}\text { Penicillin } \\
\text { antibiotics }\end{array}$ & $\begin{array}{l}\text { Amoxicillin sulbactam sodium (Shusalin) (Xie et al.,2004), } \\
\text { nafcillin sodium(Jin et al., 2004), piperacillin and } \\
\text { sulbactam (Baiding) (Zhao et al.,2009) }\end{array}$ & 9 & 10.59 & 3 & 6.00 \\
\hline $\begin{array}{l}\text { Cephalosporin } \\
\text { antibiotics }\end{array}$ & $\begin{array}{l}\text { Cefoperazone sulbactam (Sulperazon) (Zhao et al.,2009), } \\
\text { cefpiramide (Tailixin) and cefpiramide (Lixianni) (Chinese } \\
\text { Pharmacopoeia Commission., 2001), Cefaclor dispersible } \\
\text { tablets (Hengyun) (Jin et al., 2004), cefuroxime sodium } \\
\text { (Fulexin) (Jin et al., 2004), cefuroxime sodium (Andeqing) } \\
\text { (Zhao et al.,2009), ceftriaxone (Fanshengshufu) (Xie et } \\
\text { al.,2004), cefathiamidine (Xianlisu) (Jin et al., 2004) }\end{array}$ & 18 & 21.18 & 8 & 16.00 \\
\hline $\begin{array}{l}\text { Macrolide } \\
\text { antibiotics }\end{array}$ & $\begin{array}{l}\text { Azithromycin (Qixian) (Xie et al., 2004), roxithromycin } \\
\text { capsule (Zhao et al.,2009) }\end{array}$ & 6 & 7.06 & 2 & 4.00 \\
\hline $\begin{array}{l}\text { Lincosamide } \\
\text { antibiotics }\end{array}$ & $\begin{array}{l}\text { Clindamycin phosphate (Kelinmei) (Chinese } \\
\text { Pharmacopoeia Commission., 2001), clindamycin } \\
\text { phosphate (Kaifufei) (Zhao et al.,2009) }\end{array}$ & 5 & 5,88 & 2 & 4.00 \\
\hline $\begin{array}{l}\text { Fluoroquinolone } \\
\text { antibiotics }\end{array}$ & $\begin{array}{l}\text { Gatifloxacin (Haichao) (Xie et al., 2004), gatifloxacin } \\
\text { sodium chloride (Lepai) (Zhao et al.,2009), levofloxacin } \\
\text { (Zuoke) (Jian et al.,2003), levofloxacin (Hengao) (Jin et } \\
\text { al., 2004), sparfloxacin (Libeier) (Jin et al., 2004) }\end{array}$ & 13 & 15.3 & 5 & 10.00 \\
\hline Other antibiotics & $\begin{array}{l}\text { Vancomycin (Jin et al., 2004), amphotericin B liposome } \\
\text { (Amphotec) (Jin et al., 2004), amphotericin B (Jin et al., } \\
\text { 2004), rifamycin (Lifuxin) (Jin et al., 2004) }\end{array}$ & 4 & 4,71 & 4 & 8.00 \\
\hline $\begin{array}{l}\text { Blood system } \\
\text { drugs }\end{array}$ & $\begin{array}{l}\text { Carbazochrome sodium sulfonate (Jin et al., 2004), } \\
\text { granulocyte stimulating factor (Jiexin) (Jin et al., 2004) }\end{array}$ & 2 & 2,35 & 2 & 4.00 \\
\hline
\end{tabular}




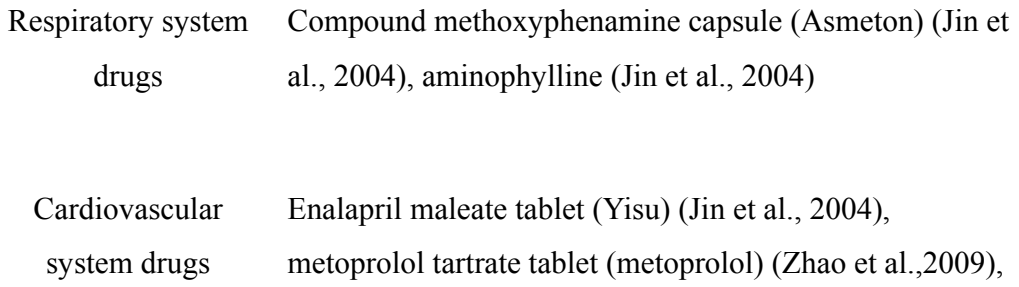

Analgesic-antipyre Celecoxib (Celebrex) (Jin et al., 2004) tic drugs

Other drugs Compound water-soluble vitamin (Jin et al., 2004), Shuxuetong (Jin et al., 2004), Gushukang particles (Jin et al., 2004), Xiyanping (Zhao et al.,2009), magnesium isoglycyrrhizinate (Jin et al., 2004), compound meglumine diatrizoate injection (Jin et al., 2004), sodium aescinate for injection (Zhao et al.,2009), propofol injection (Jin et al., 2004), lentinan (Jin et al., 2004), fructose diphosphate injection (Jin et al., 2004), Zhikang capsule (Jin et al., 2004), Panlongqi tablet (Jin et al., 2004)

by year, and target organs show high sensitivity to drugs, leading to enhanced drug action intensity and prolonged action time; (2) in the elderly, liver and kidney volume gradually decrease, blood flow relatively decreases, liver and kidney blood flow is only about half of a strong man, its metabolic capacity is very low, the first-pass effect is gradually weakened, the bioavailability relatively increases, drug metabolism slows down, and drug half-life is relatively prolonged; (3) the elderly usually have many chronic diseases, concomitant medication is common, and drug interaction and other reasons cause poor medication compliance; (4) because of weakened body functions in the elderly, the ability to digest and absorb drug orally administrated is low, the volume distribution for target organs of fat-soluble drugs increases, albumin in plasma reduces, protein binding of the drugs with high protein binding rate drops sharply, and free drug concentration body increases, resulting in more prone to poisoning and adverse reactions than the youth. Therefore, hospital doctors should choose drugs cautiously according to the special physiological and pathological characteristics of the elderly, adhere to the principles of 
http://dx.doi.org/10.4314/ajtcam.v10i3.18

rational drug use, regulate clinical drug dosage, achieve individualized and personalized drug administration, and strengthen department monitoring during drug administration process, so as to effectively reduce the incidence of ADR.

\section{Relationship between family allergic history and past ADR history and ADR}

It can be seen from Table 2 that unknown family history and past history account for $54.12 \%$ and $60.00 \%$ respectively, indicating that a large proportion of patients did not pay sufficient attention to the family and past ADR history. In daily clinical work in hospital, paying attention to past allergic history or ADR history will improve drug use safety factor, and emphasize on preventive and protective measures to reduce the risk of drug use.

\section{Relationship between drug administration routes and ADR}

It can be seen form Table 3 that in the 85 cases of ADR, 73 cases were caused by intravenous administration, indicating that ADR caused by intravenous administration route is much higher than other routes, because the drug administration route of intravenous infusion is taken as the main treatment method in inpatients. At intravenous infusion, drugs directly enter human circulation, with fast action and no first-pass effect, the pharmacological effects and adverse reactions are stronger than oral administration, and many factors induce ADR, including $\mathrm{pH}$ value, osmotic pressure, temperature, particulates, creotoxin, etc (Ling et al., 2005). The requirements of intravenous administration are much higher than other preparations, so improper preparation operation, low preparation quality, inappropriate drug combination, long-term preparation liquid placing, high impurity content and fast (low) infusion will cause ADR. Therefore, at clinical drug administration, doctors should strictly ask patient's medication history, try to minimize intravenous administration, and adopt oral administration or intramuscular injection instead of intravenous administration if possible (Wang et al., 2009; Zhao et al., 2009). In case of special demand for intravenous administration, drug administration speed should be controlled, especially for certain stimulant drugs.

\section{Relationship between drug combination and ADR}

Drug combination always causes drug interactions, and more drugs are combined, the higher the incidence of ADR is. Drug combination is an important cause of ADR, and the combination of antimicrobial drugs with other drugs can induce or aggravate adverse drug reactions (Wu et al., 2010). Therefore, it is suggested to minimize the drug varieties needed for clinical treatment.

\section{Relationship between drugs and ADR}

It can be seen from Table 4 that the 85 cases of ADR involve a total of 50 drugs in 12 categories, in which, the most involved is antimicrobial drugs, whether the varieties of ADR caused or the number the cases rank the first, consistent with the similar domestic report result (Yang et al., 2001). This may be related with gradually increased frequency and dosage of clinical use of antimicrobial drugs, increased bacterial resistance, non-indicative drug use, drug combination, overdose, long drug use time, etc. Irrational use of antibiotics not only increases the economic burden of patients, and also increases the incidence of ADR, as well as causes bacterial resistance, increases associated complications and prolongs the course of disease. Therefore, doctors need focus on the rational use of antibiotics and the scope of application, and the antibiotics causing ADR are mostly cephalosporins and fluoroquinolones. Cephalosporins have broad antimicrobial spectrum, obvious bactericidal effects, stable action on $\beta$-lactamase, relatively low toxic reactions and good clinical efficacy and are very 
commonly used in clinical conditions, so the ADR cases are more reported. This should remind us that doctors should pay full attention to ADR caused by cephalosporins. The clinical manifestations are mainly allergic-like reactions and involve many organs and systems, so clinicians should use antibiotics in strict accordance with the "Guiding principles for clinical use of antibiotics", and it is suggested to make skin test when cephalosporins use is indicated, to reduce the incidence of adverse reactions (Jin et al., 2004).

\section{Relationship between clinical manifestations and ADR}

It can be seen from Table 5 that the clinical manifestations of ADR are mostly skin and accessory damage and secondly digestive system and nervous system damages, however, it is easy to find and diagnose these two reactions in clinical conditions. The incidence of allergic skin reaction is high, but the majority of disease conditions are mild, and the symptoms will disappear immediately after drug withdrawal or giving antihistamines. In addition, some commonly used drugs in clinical use, such as traditional Chinese medicine compound, anti-infective drugs, etc, are antigens, easy to cause allergic reactions after entering into body, so as to cause dermatitis, rash and other symptoms (Zhang et al., 2010).

\section{Conclusion}

It is impossible for completely prevent ADR, but it is possible to reduce the incidence of ADR. In clinical drug use, the following can be adopted to reduce the incidence of ADR: (1) Pay attention to patient's constitution and past medical history; to be cautious to use drugs in the elderly and children and strictly master the indications and dosage. (2) Pay attention to drug administration methods and compatibility: to minimize the proportion of intravenous administration and combination of many drugs. (3) Strengthen the rational use of anti-infective drugs: to reduce or avoid long drug use, many combined drugs and long course of treatment. (4) Strengthen ADR event reporting and feedback, provide and perfect drug safety data, and avoid or reduce potential ADR.

Adverse drug reaction is very harmful, and it increases medical costs and brings big economic losses, and in severe cases, causes deformity, cancer and mutation and even endangers the life of patients, therefore, medical care staff must pay high attention to them. Hospital administration departments should pay full attention to the service functions of clinical pharmacist, and pharmacists should cooperate with physicians to make rational drug use regimens and actively carry out the following responsibilities in clinical monitoring of ADR: (1) to help doctors find ADR events in time and accurately fill in the relevant reports; (2) to collect and assort ADR information in the whole hospital in certain period, timely feedback to clinicians, and increase the warning capability for the drugs causing ADR; (3) to monitor drug use in special populations, such as patients with liver and kidney dysfunction, infants and children, elderly patients and breastfeeding women; (4) to release timely ADR information to clinical professional journals and improve preventive consciousness to clinical drug-induced diseases.

\section{References}

1. (2001). Chinese Pharmacopoeia Commission. Pharmacopoeia of the People's Republic of China - Notices on clinical drug use. Version 2. Beijing: Chemical Industry Press, Catalogue.

2. Jian Y J,Wang Y Q,Chang H J,et al. (2003). Analysis of adverse effects associated with traditional Chinese medicines in 1230 cases. Adverse Drug React J, 5(4): 231-234.

3. Jin Guilan, Tang Wen, Zhao Meiju. (2004). Analysis on 528 case reports of adverse drug reactions. Chinese Journal of Pharmacoepidemiology, 13(5): 247-248. 
4. Jin Guilan, Tang Wen, Zhao Meiju. (2004). Analysis on 528 case reports of adverse drug reactions. Chinese Journal of Pharmacoepidemiology, 13(5): 247-248.

5. Ling C Y, Zhang J P, Ge W H. (2005). Analysis of 242 ADR cases in our hospital. China Pharm, 16(9): 455-456.

6. Wang Ruicai, Wang Shuqing. (2009). Cautious intravenous infusion according to disease conditions. Modern Journal of Integrated Traditional Chinese and Western Medicine, 18 (13): 1526-1527.

7. Wang Wei. (2009). Reasons of adverse reactions caused by Chinese medicine injections and preventive measures. Guide of China Medicine, 7 (2): 63-64.

8. Wu C S, Zhang Z W. (2010). Analysis of adverse effects associated with traditional Chinese medicines in 77 cases. Asia-Pacific Traditional Medicine, 5(6): 162-164.

9. Xie Jinzhou. (2004). Adverse drug reactions and monitoring. Beijing: China Medical Science Press, : 52.

10. Yang X H. (2001). Analysis of 141 cases of developing adverse reactions to antibiotics. China Pharm, 12(2): 106-107.

11. Zhang Yunlin. (2010). Analysis on 521 case reports of adverse drug reactions. Journal of Hunan Medical College, 16(4): 515 .

12. Zhao Quan, Liang Yanping, Cheng Dongsheng, et al. (2009). Analysis on 770 case reports of adverse drug reactions. Chinese Journal of Hospital Pharmacy, 29(19): 1694.

13. Zhao Baoxia, Guo Hui. (2009). Analysis on 285 cases of adverse drug reactions and nursing measures. Journal of Clinical Medicine in Practice, 5 (1): 33-34. 